\title{
Electronic and Optical Properties of Quantum Dot Surrounded by Doped Cylindrical Nanowire
}

\author{
MARYAM MORADi ${ }^{a}$, MAHMOOD MORADI $^{b, *}$, \\ S.M. Elahi ${ }^{a}$ AND S.S. PARHIZGAR ${ }^{a}$ \\ ${ }^{a}$ Department of Physics, Faculty of Sciences, Science and Research Branch, \\ Islamic Azad University, Tehran, Iran \\ ${ }^{b}$ Department of Physics, College of Sciences, Shiraz University, Shiraz 71946-84795, Iran
}

Received: 19.05.2020 \& Accepted: 30.06.2020

Doi: $10.12693 /$ APhysPolA.138.561

*e-mail: mmoradi@shirazu.ac.ir

\begin{abstract}
The electronic and optical properties of InAs spherical quantum dot (QD) at the center of a doped $n$-type GaAs cylindrical nanowire were investigated by solving the Schrödinger and Poisson equations self consistently. The effect of donor density of a cylinder and the size of QD on the allowed energy levels, electrostatic potential, conduction bands and transition energy of the system were calculated. The optical properties related to intra-band transitions in the conduction band of this structure were studied through the compact density matrix approach. The results showed that the peak position and magnitude of optical absorption coefficients, which are mainly located in the far infrared region, and the refractive index changes were significantly affected by the incident optical intensity and the doping concentration of the surrounding media
\end{abstract}

topics: $n$-type doping, cylindrical nanowire, spherical quantum dots, self-consistent Schrödinger-Poisson

\section{Introduction}

Based on semiconductor nanowire properties, model devices and applications have been arranged in the fields of electronics, photonics, mechanics and sensors [1]. Single photon sources are one of the main components of quantum photonic applications. These sources, which ideally emit a single photon at a time, are highly efficient and can be integrated into photon circuits for the design of complex quantum systems. Various systems have been actively studied to obtain such resources, including semiconductor quantum dots (QDs). The quantum dots embedded in the III-V compound semiconductor nanowires perform relatively well and are flexibly constructed and integrated [2]. The growth processes of nanowires begin to be developed as the embedded QD which provides a means for QDs to be completely positioned [3, 4]. In addition, the embedded QDs are often used to increase the emission and extraction of light in nanophotonic structures, for example with nanowires acting as photonic structures that enhance light extraction $[5,6]$. The InAs QDs have a unique quantum efficiency while growing in the GaAs matrix [7]. Changes in multidimensional and multistructural QD parameters have already been studied [8]. The effect of size change on the work function of InAs quantum dots grown on the GaAs matrix was studied experimentally [9] and it was observed that the charge accumulation is affected by the size of quantum dots.
Quantum dots can theoretically provide flexibility of narrow or symmetrical emission spectra for optical applications. Furthermore, since the electronic properties control many other features of dots, it is vital to connect different optical spectroscopies to the electronic characteristics of QDs. As expected, the transition energy of quantum dots is negatively correlated with the volumetric size for each geometry. Moreover, the spherical quantum dots have significantly higher values of transition energy than cubic quantum dots with the same real space, because the spherical quantum dots have a higher symmetry, which reinforces their quantum confinement effects $[10,11]$.

Introducing impurity into the lattice allows quantum dots' electronic properties to be tailored and customized to the engineering need. The wave functions and energy levels depend on the amount and location of impurities. However, the use of real devices in electronics and photonics requires the effective and controlled doping of semiconductors to modify their electrical, optical and magnetic properties [1]. In principle, it is possible to insert active dopants into nanowires in two ways, namely doping during the growth process and doping after growth through ion diffusion or implantation [12]. Consequently, it is necessary to carry out reliable and controlled systematic doping studies of semiconductor nanowires, placing more emphasis on measuring the type of doping and transport characteristics for nanostructures in the future [13]. 
The properties of $\mathrm{GaAs} / \mathrm{Ga}_{1-x} \mathrm{Al}_{x} \mathrm{As}$ heterojunction superlattice, in which only the $\mathrm{Ga}_{1-x} \mathrm{Al}_{x} \mathrm{As}$ layers are doped with $\mathrm{Si}$ impurities, were reported in [14]. Next, the epitaxial growth was developed to fabricate the successive controlled layers of different semiconductors to gain high electrical mobility and fast operation [15]. Further, a doped layer was added to the desired structures during the epitaxial growth of a layer, e.g. the effect of a magnetic field on the electronic structure of a selectively doped single quantum well of $\mathrm{Al}_{x} \mathrm{Ga}_{1-x} \mathrm{As} / \mathrm{GaAs}$ was measured [16]. Afterwards, the electronic subband structure in $\mathrm{Al}_{x} \mathrm{Ga}_{1-x} \mathrm{As} / \mathrm{GaAs}$ superlattice was calculated self consistently through the Schrödinger and Poisson equations as suggested in [17], and the effects of parameters such as the donor concentration, well width and barrier width were investigated. Finally, the electron dynamics in $p$-doped self-assembled InGaAs/GaAs quantum dots at low temperature and at room temperature were assessed and the rise times of the structure were compared with those of the undoped quantum dots [18].

West et al. [19] reported the experimental results for inter-band transition between two of the lowest levels (the ground and first excited states) of doped GaAs quantum well. The change of doping concentration and electric field affect the confining potential, sub-band structure, optical transition and nonlinear optical properties in a modulation doped of $\mathrm{GaAs} / \mathrm{Ga}_{1-x} \mathrm{Al}_{x} \mathrm{As}$ double well [20-22]. The energy eigenvalues and the wave functions of the three lowest levels (the ground and first two excited states) in a rectangular GaAs quantum dot in the presence of donor impurity, adopting the quasi one-dimensional effective potential model and effective mass approximation, were investigated. They obtained electric fields to study the effects of these parameters on the optical properties of the structure [23]. The electronic properties of asymmetric double delta doped wells (GaAs) were calculated by a self-consistent solution of Schrödinger-Poisson equation to study the effects of the structure parameters on these properties and also the nonlinear second order transitions between the subbands [24]. Elsewhere, the inter sub-band optical transition in ultrathin InAs impurity layers, embedded in the bulk InP semiconductors quantum well, was reported [25]. Further, it was reported that the optical properties are significantly affected by quantum confinement [26, 27]. Likewise, the optical properties such as absorption coefficients and refractive index changes of the excitonic transition $1 s-1 p$ in the spherical quantum dot core/shell (AlN/GeN), were studied [28]. Also, the linear and nonlinear optical properties of a confined exciton in spherical quantum dots (GaAs) and the effect of temperature and pressure on these parameters were investigated $[29,30]$. The optical properties related to the inter-sub band optical transitions for direct bandgap GeSn/Ge quantum dots are obtained numerically by Baira et al. [31]. The effects of important factors such as strength and doping position on linear and nonlinear optical properties of the GaAS/AlGaAs multiple quantum well within the effective mass approximation were also investigated [32]. Moreover, the influence of bismuth and doping type on the optical properties of $n$-type and p-type $\mathrm{GaAsBi} / \mathrm{AlGaAs}$ single quantum well heterostructures were investigated, the results of which were compared with those of the $n$-type and $p$-type GaAs/AlGaAs quantum well structures [33].

The electronic and optical properties of a spherical quantum dot at the center of a cylindrical nanowire system were investigated in $[34,35]$. In those papers a single-band and constant confining potential were used in order to determine the electronic structure [34], theoretically achieved by solving the Schrödinger equation alone. For complex systems, the electrostatic potential of the structure cannot be considered as a constant, which becomes even more obvious when there are impurities and thereby free charges in the structure. In fact, the effects of free carrier and accumulation of charge in the structure cannot be ignored. Therefore, in this study, we particularly developed the effect of doping of the surrounding media, the cylindrical nanowire, on QD by introducing the selfconsistent coupled Schrödinger-Poisson equations to calculate the physical quantities of the nanostructures more precisely.

The rest of this article is organized as follows: first, the basic equations and the coupled Schrödinger-Poisson equations are presented. Further, the related equations are written in cylindrical coordinates as it is required in our calculations and some optical parameters are introduced. The selfconsistency requirement in doped heterostructures is an aspect of the calculations of electronic properties [36]. Next, the aforementioned equations are used to consider the electronic and optical properties of a quantum dot located at the center of a doped cylindrical nanowire. Finally, the effects of the doping of the nanowire on the physical properties of QD are determined and ways how this impurity can affect physical properties are explained.

\section{Basic equations}

It is necessary to solve the Schrödinger equation along with the Poisson equation simultaneously to obtain electronic properties of materials acurately, in particular the nanostructures at different physical conditions. This gives relatively precise results as it takes into account the interaction of electrons and electric potentials at each point. The Hamiltonian of a crystalline solid system consisting of electrons and ions is given by:

$$
-\nabla \cdot\left(\frac{\hbar^{2}}{2 m_{e}^{*}} \nabla \psi_{e}(\boldsymbol{r})\right)+V(\boldsymbol{r}) \psi_{e}(\boldsymbol{r})=E_{e} \psi_{e}(\boldsymbol{r}) .
$$

Here, $m_{e}^{*}$ is the effective mass of the electron, $\hbar$ is Planck's constant, $E_{e}$ and $\psi_{e}(\boldsymbol{r})$ are energy eigenvalue and wave function of the conduction band, 
respectively, and $V(\boldsymbol{r})$ is the spatially varying potential. In order to obtain the self-consistent solution of the structure, the Poisson equation must be solved to determine the electrostatic potential. The Poisson equation links the charge density distribution and the electrostatic potential of the structure and is given by:

$$
\boldsymbol{\nabla} \cdot[\varepsilon \nabla \phi(\boldsymbol{r})]=\tilde{\rho}(\boldsymbol{r}),
$$

where

$$
\tilde{\rho}(\boldsymbol{r})=-e\left(N_{d}^{+}-n_{e}\right) .
$$

Here, $\varepsilon$ is the static dielectric constant, $e$ is the electron charge, $N_{d}^{+}$is the total density of ionized dopants and $n_{e}$ is the electron density distribution in the conduction band. The electrostatic potential $\phi(\boldsymbol{r})$ in (2) is related to the potential energy $V(\boldsymbol{r})$ in an arbitrary quantum well as follows:

$$
V(\boldsymbol{r})=-e \phi(\boldsymbol{r})+\Delta E_{c},
$$

where $\Delta E_{c}$ is due to the heterojunction band offset [37]. The electron density $n_{e}$ is calculated with

$$
n_{e}=\sum_{m} n_{m}\left|\left(\psi_{e}\right)_{m}\right|^{2}
$$

where $m$ is the number of a bound state, and $n_{m}$ is the number of electron occupation in the $m$-th subbands of the wave function $\psi_{e}$ [22]. To calculate $n_{m}$, one should integrate the density of the states $D(E)$ weighted by the Fermi Dirac distribution function:

$$
f(E)=\frac{1}{1+\exp \left(\frac{E-E_{f}}{k_{\mathrm{B}} T}\right)},
$$

accordingly

$$
n_{m}=\int_{E_{m}}^{\infty} \frac{D(E)}{1+\exp \left(\frac{E-E_{f}}{k_{\mathrm{B}} T}\right)} \mathrm{d} E .
$$

Here $T$ is the selected temperature for selfconsistent calculation $(T=300 \mathrm{~K}), E_{f}$ is the Fermi energy, $E_{m}$ is the subband energy level that is occupied by an electron and $k_{\mathrm{B}}$ is the Boltzmann constant.

\section{Self-consistent solution of Schrödinger-Poisson equations}

The self-consistent solution involves solving the Poisson equation to achieve the electric potential related to the distribution of charge carriers and donor atoms. By using this electric potential in the Schrödinger equation, the eigenvalues and associated eigenfunctions are obtained, and subsequently the new charge distribution is calculated. The process of calculating the electric potential from the charge distribution and then calculating the charge distribution from the electric potential is repeated through iteration until the electric potential satisfies certain error criteria. In order to solve these equations numerically, it is necessary to discretize the Schrödinger and Poisson equations by a finite difference scheme to obtain a standard matrix eigenvalue problem.
The Poisson equation can be written in cylindrical coordinates. Because of the axial symmetry in the structure, the azimuthal angle dependency is ignored. The equation governing the potential is as follows:

$$
\left(\frac{\partial^{2}}{\partial \rho^{2}}+\frac{1}{\rho} \frac{\partial}{\partial \rho}+\frac{\partial^{2}}{\partial z^{2}}\right) \phi=-\frac{\tilde{\rho}(\rho, z)}{\varepsilon} .
$$

This equation is discretized by using the finite difference three-point scheme:

$$
\phi_{i, j}=\frac{1}{\Sigma_{i, j}}\left[\frac{\rho_{i, j}}{\varepsilon_{i}}+A_{i, j}\right],
$$

where

$$
\begin{aligned}
A_{i, j} & =\phi_{i, j-1}\left(\frac{1}{\Delta \rho^{2}}-\frac{1}{2 \rho_{i} \Delta \rho}\right) \\
+\phi_{i, j+1}\left(\frac{1}{\Delta \rho^{2}}+\frac{1}{2 \rho_{i} \Delta \rho}\right) & \\
\Sigma_{i, j} & =\frac{2}{\Delta \rho^{2}+\Delta z^{2}} .
\end{aligned}
$$

In the above relations, the index $i$ indicates the mesh points in the $z$-direction and index $j$ shows the mesh points along the $\rho$-direction, $\Delta z$ and $\Delta \rho$ also denote the mesh size between two points in the $z$ and $\rho$-direction, respectively. Due to cylindrical symmetry (axial symmetry), the azimuthal part of wave functions is separated as $\Phi(\varphi)=\frac{1}{\sqrt{2 \pi}} \mathrm{e}^{\mathrm{i} m \varphi}$, with $m=0, \pm 1, \pm 2, \pm 3, \ldots$ For the ground state wave function, the azimuthal quantum number is zero $(m=0)$. Therefore, the Schrödinger equation can be rewritten in cylindrical coordinates as:

$$
\begin{aligned}
& -\left[\frac{\hbar^{2}}{2 m_{e, h}^{*}}\left(\frac{\partial^{2}}{\partial \rho^{2}}+\frac{1}{\rho} \frac{\partial}{\partial \rho}+\frac{\partial^{2}}{\partial z^{2}}\right)+V(\rho, z)\right] \\
& \quad \times \psi_{n}^{e, h}(\rho, z)=E_{n}^{e, h} \psi_{n}^{e, h}(\rho, z)
\end{aligned}
$$

and further also discretized, using a three-point finite difference scheme, namely:

$$
\begin{aligned}
& -\frac{\hbar^{2}}{2 m_{e, h}^{*}}\left[\frac{1}{\Delta \rho^{2}}\left(\psi_{i+1, j}-2 \psi_{i, j}+\psi_{i-1, j}\right)\right. \\
& +\frac{1}{2(i-1) \Delta \rho}\left(\psi_{i+1, j}-\psi_{i-1, j}\right) \\
& \left.\quad+\frac{1}{\Delta z^{2}}\left(\psi_{i, j+1}-2 \psi_{i, j}+\psi_{i, j-1}\right)\right] \\
& +V_{i, j} \psi_{i, j}=E \psi_{i, j} .
\end{aligned}
$$

This is a differential equation that connects the value of the wave function at the grid point $\left(\rho_{i}, z_{i}\right)$ to its values at the neighboring grid points $\left(\rho_{i \pm 1}, z_{i}\right)$ and $\left(\rho_{i}, z_{i \pm 1}\right)$. The potential energy term $\left(V_{i, j}\right)$ in this equation is obtained by discretizing (4) as follows:

$$
V_{i, j}=-q \phi_{i, j}+\Delta E_{c_{i, j}},
$$

where $\phi_{i, j}$ is the solution of (9).

\section{Optical properties}

When the electronic structures such as energies and corresponding wave functions are determined, it is appropriate to calculate the optical properties 
such as the absorption coefficients (ACs) and refractive index (RI) changes for the structure, using the density matrix approach. These coefficients are calculated $[38,39]$ for transition between the ground and first excited states. The linear and

third order nonlinear absorption coefficients are given by:

$$
\alpha^{(1)}(\omega)=\omega \sqrt{\frac{\mu}{\varepsilon_{r} \varepsilon_{0}}} \frac{\sigma_{v}\left|M_{12}\right|^{2} \hbar \Gamma_{12}}{\left(E_{21}-\hbar \omega\right)^{2}+\left(\hbar \Gamma_{12}\right)^{2}}
$$

and

$$
\begin{aligned}
& \alpha^{(3)}(I, \omega)=-\omega \sqrt{\frac{\mu}{\varepsilon_{r} \varepsilon_{0}}} \frac{I}{2 \varepsilon_{0} n_{r} c} \frac{\sigma_{v}\left|M_{12}\right|^{2} \hbar \Gamma_{12}}{\left[\left(E_{21}-\hbar \omega\right)^{2}+\left(\hbar \Gamma_{12}\right)^{2}\right]^{2}} \\
& \quad \times\left\{4\left|M_{12}\right|^{2}-\frac{\left(M_{22}-M_{11}\right)^{2}\left[3 E_{21}^{2}-4 E_{21} \hbar \omega+\hbar^{2}\left(\omega^{2}-\Gamma_{12}^{2}\right)\right]}{E_{21}^{2}+\left(\hbar \Gamma_{12}\right)^{2}}\right\}
\end{aligned}
$$

and for the total ACs, the expression:

$$
\alpha(I, \omega)=\alpha^{(1)}(\omega)+\alpha^{(3)}(I, \omega)
$$

is defined.

The linear and nonlinear RI changes can be obtained by the same procedure, thus:

$$
\frac{\Delta n^{(1)}(\omega)}{n(r)}=\omega \frac{1}{2 \varepsilon_{0} n_{r}^{2}} \frac{\sigma_{v}\left|M_{12}\right|^{2}\left(E_{21}-\hbar \omega\right)}{\left(E_{21}-\hbar \omega\right)^{2}+\left(\hbar \Gamma_{12}\right)^{2}}
$$

and

$$
\begin{aligned}
& \frac{\Delta n^{(3)}(\omega)}{n(r)}=\frac{\mu c I}{4 n_{r}^{3} \varepsilon_{0}} \frac{\sigma_{v}\left|M_{12}\right|^{2}}{\left[\left(E_{21}-\hbar \omega\right)^{2}+\left(\hbar \Gamma_{12}\right)^{2}\right]^{2}} \\
& \quad \times\left\{4\left|M_{12}\right|^{2}\left(E_{21}-\hbar \omega\right)-\frac{\left(M_{22}-M_{11}\right)^{2}}{E_{21}^{2}+\left(\hbar \Gamma_{12}\right)^{2}}\right. \\
& \left.\quad \times\left[\left(E_{21}-\hbar \omega\right)\left(E_{21}\left(E_{21}-\hbar \omega\right)-\left(\hbar \Gamma_{12}\right)^{2}\right)-\left(\hbar \Gamma_{12}\right)^{2}\left(2 E_{21}-\hbar \omega\right)\right]\right\}
\end{aligned}
$$

and the total RI changes can be expressed as:

$$
\frac{\Delta n(\omega, I)}{n(r)}=\frac{\Delta n^{(1)}(\omega)}{n(r)}+\frac{\Delta n^{(3)}(\omega, I)}{n(r)} .
$$

In (15)-(19), $I=2 \varepsilon_{0} n_{r} c|E|^{2}$ is the intensity of the electromagnetic field, $E_{21}=E_{2}-E_{1}$ is the energy difference between the two lowest electronic states and $\sigma_{v}$ represents the electron density. Next, $\mu$ and $\varepsilon_{r}$ are the permeability and permittivity of the system, respectively, and $\Gamma_{12}=\frac{1}{\tau}$, where $\tau$ is the relaxation time and $M_{i j}=\left|\left\langle\psi_{i}|e z| \psi_{j}\right\rangle\right|$, where $(i j=1,2)$ are the matrix elements of the electric dipole moment which represents the overlap between the wave functions and the polarization vector along the $z$ axis. These elements depend on the polarity of the electromagnetic field, and here only the third component appears in the calculations because the polarity of the field is in the direction of the $z$ axis. It should be noted that due to the axial symmetry of the structure, even if the electromagnetic field along the radial axis is also considered, the contribution of the other components of the electric dipole moment is almost zero.

\section{Results and discussion}

To investigate the accuracy and reliability of the present method, we first consider a cylindrical GaAs nanowire with length $L=400 \mathrm{~nm}$ and radius
$R_{2}=20 \mathrm{~nm}$. The numerical calculated eigenenergies (the ground state and first excited energies) are given in Table I and compared with the analytical result given by:

$$
E_{n, m, i}=\frac{\hbar^{2}}{2 m}\left[\left(\frac{\alpha_{m, i}}{R}\right)^{2}+\left(\frac{n \pi}{l}\right)^{2}\right] .
$$

The parameter $\alpha_{m, i}$ is the $i$-th root of the Bessel function, i.e., $j_{m}\left(\alpha_{m, i}\right)=0$. Here, we use the same parameters as those previously used in [40]. The outcome shows that the numerical results are in good agreement with analytical solutions.

In the following, we consider a spherical InAs quantum dot embedded in the center of a GaAs cylindrical nanowire (see Fig. 1). It is assumed that the nanowire material with a wider gap is uniformly doped with $n$-type but the quantum dot material with a narrower gap is undoped. Also, the strain effects are neglected just for simplicity reasons. The electronic properties of the structure are calculated, using the discretized self-consistent solution of the coupled Schrödinger-Poisson equations, namely (9) and (13), as well as the optical properties are obtained using the compact density matrix approach, namely (15)-(20).

According to Fig. 1, the radius of quantum dot is $R_{1}$ and the radius and height of cylindrical nanowire are $R_{2}$ and $L$. In all calculations, it is assumed 
TABLE I

Comparison between analytical and numerical values (this work) for ground state and first excited states of different carriers in a cylindrical GaAs nanowire.

\begin{tabular}{c|c|c|c|c}
\hline \hline \multirow{2}{*}{$\mathrm{N}$} & \multicolumn{2}{|c|}{ Analytical solution } & \multicolumn{2}{c}{ Numerical solution (this work) } \\
\cline { 2 - 5 } & Electron $[\mathrm{meV}]$ & Heavy hole $[\mathrm{meV}]$ & Electron / error [meV\%] & Heavy hole / error [meV\%] \\
\hline 1 & 463.2678 & 318.3378 & $466.51067 / 0.7 \%$ & $318.9745 / 0.2 \%$ \\
2 & 463.3031 & 318.3440 & $466.6146 / 0.715 \%$ & $318.9928 / 0.2 \%$
\end{tabular}

TABLE II

The depletion region, energy of ground state and first excited state of electron in the conduction band of QD and their differences for different donor concentrations.

\begin{tabular}{c|c|c|c|c}
\hline \hline $\begin{array}{c}\text { Donor density } \\
\times 10^{18}\left[\mathrm{~cm}^{-3}\right]\end{array}$ & $\begin{array}{c}\text { Depletion } \\
\text { region }[\AA]\end{array}$ & $\begin{array}{c}\text { Ground state bound } \\
\text { in QD }[\mathrm{meV}]\end{array}$ & $\begin{array}{c}\text { First excited state } \\
\text { bound in QD }[\mathrm{meV}]\end{array}$ & $E_{21}[\mathrm{meV}]$ \\
\hline no impurities & - & 15.6 & 94.4 & 78.8 \\
0.01 & 160 & -80 & -7.5 & 74 \\
0.05 & 125 & -200 & -134 & 66 \\
0.1 & 85 & -251.6 & -188.8 & 62.8 \\
0.5 & 35 & -394 & -339.4 & 54.6 \\
1 & 23 & -451.3 & -398.5 & 52.8
\end{tabular}

that $R_{1}=10 \mathrm{~nm}, R_{2}=20 \mathrm{~nm}$, and $L=400 \mathrm{~nm}$. Moreover, we took $\sigma_{v}=10^{24} \mathrm{~m}^{-3}, T=300 \mathrm{~K}$, $\Gamma_{12}=\frac{1}{\tau_{12}}, \tau_{12}=0.2 \mathrm{ps}$, and $\mu=4 \pi \times 10^{-7} \mathrm{H} / \mathrm{m}$. The cylindrical nanowire is uniformly doped by a donor atom in such a way that it acts as an $n$ type crystal.

In Fig. 2, the conduction band edge of the system against the distance from the center of QD along the cylinder axis ( $z$ direction) for a different donor concentration is presented. It can be observed that the width of the depletion region decreases as the donor concentration increases and the band bending near the interface becomes sharper. Inside QD, the concentration of more charges on the surface of QD or their penetration inside changes the potential energy of the surface whereby the conduction band edge inside QD decreases. Since the conduction band edge of QD is lower than that of the cylinder, the electron in the cylinder penetrates to QD. Increasing the doping concentration results in more electrons being sent to QD. The flow of charge carriers into QD is terminated when the available energy levels of QD become full and also the accumulation of charge carriers in the quantum dot produce a coulomb repulsive potential that prevents the new charges to enter QD. As a result, the potential energy inside QD increases. According to Fig. 2, the doping concentration increases and, simultaneously, so does the depth of $\mathrm{QD}$ and the width of the depletion layer narrows down. Table II shows the approximate width of the depletion regions on both sides of the quantum well, the energy of ground state, first excited state and their differences $E_{21}$ of QD. As shown in Table II, when the doping concentration increases, the energy $E_{21}$ decreses.

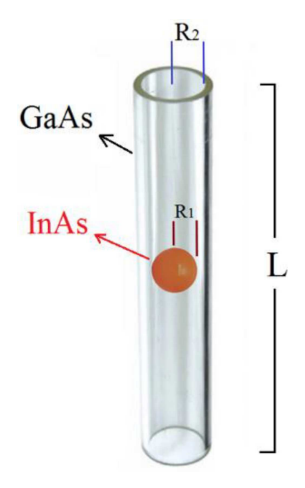

Fig. 1. The schematic diagram of an InAs spherical quantum dot located at the center of a GaAs cylindrical nanowire.

The electrostatic potential of the system is plotted for different $n$-doping concentrations at $300 \mathrm{~K}$ in Fig. 3. The doping concentration changes the electrostatic potential and creates a well there. A larger donor concentration has a narrower depletion region which results in a smaller change in the potential and lessens its depth. In the cylinder region for more doping concentration, a sharper bending at each interface is observed. This effect explains why the width of depletion is narrowing. As it is observed in Fig. 3, beyond the depletion region, the slope of the electrostatic potential turns to zero. For $N_{d}=0$, the electrostatic potential inside and outside of QD is almost constant.

As it is presented in Fig. 4, there are some extra electrons inside QD which produce a negative charge but around QD there exists a positive charge in the depletion layer which is an additional confirmation of the trend of electrostatic 


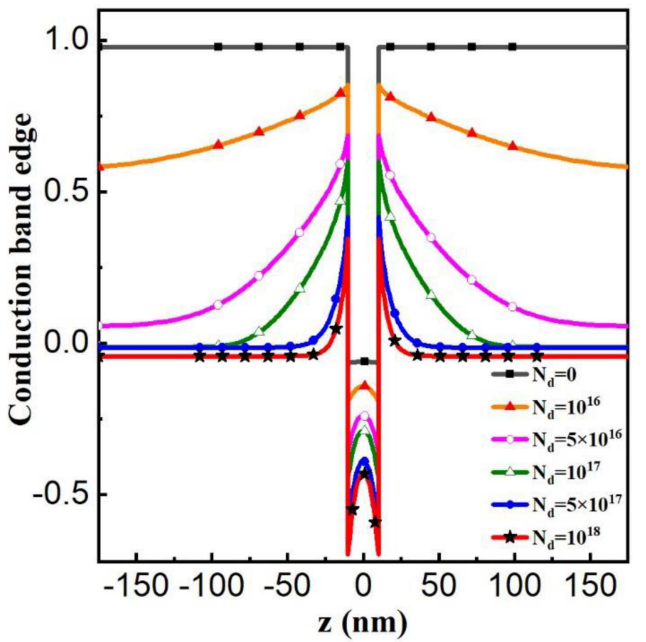

Fig. 2. The effect of $n$-type donor concentration $\left(\mathrm{cm}^{-3}\right)$ of the surrounding media on the conduction band edge of the cylinder and the spherical QD along the cylinder axis, $z$ direction, the zero point is at the center of QD.

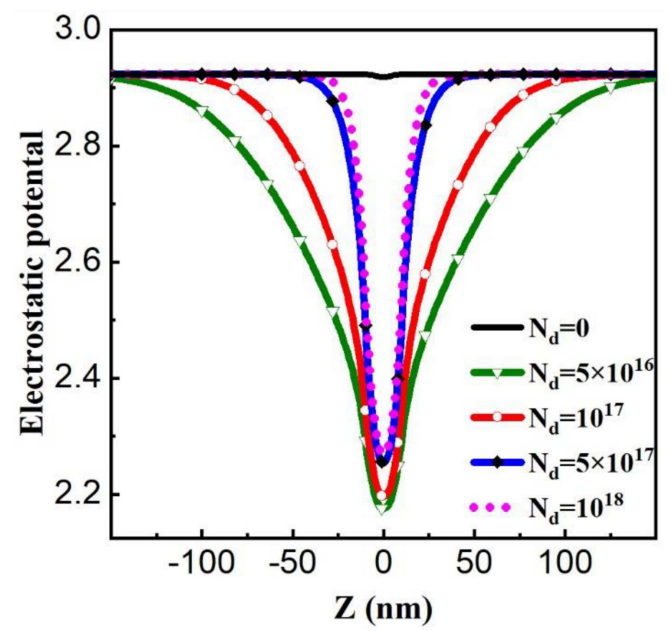

Fig. 3. The effect of donor concentration (in $\mathrm{cm}^{-3}$ ) of the cylinder on the electrostatic potential of the InAs/GaAs structure along the cylinder axis, at $T=300 \mathrm{~K}$.

potential in Fig. 3. Also, it can be seen that a wider depletion region arises from smaller donor concentration. As Fig. 4 shows, increasing the doping concentration of the cylinder boosts the positive charges around QD. In the QD region, the negative charges are concentrated and for a smaller doping concentration, the oscillation shows that there are spherical shell layers with different charge distribution which could be attributed to the repulsion behavior of the electrons.

The energy differences between the ground and first excited states of QD as a function of its radius for different donor concentrations are shown in Fig. 5. As the QD radius increases, the transition energy between the ground state and first excited state decreases. Indeed, increasing the QD size

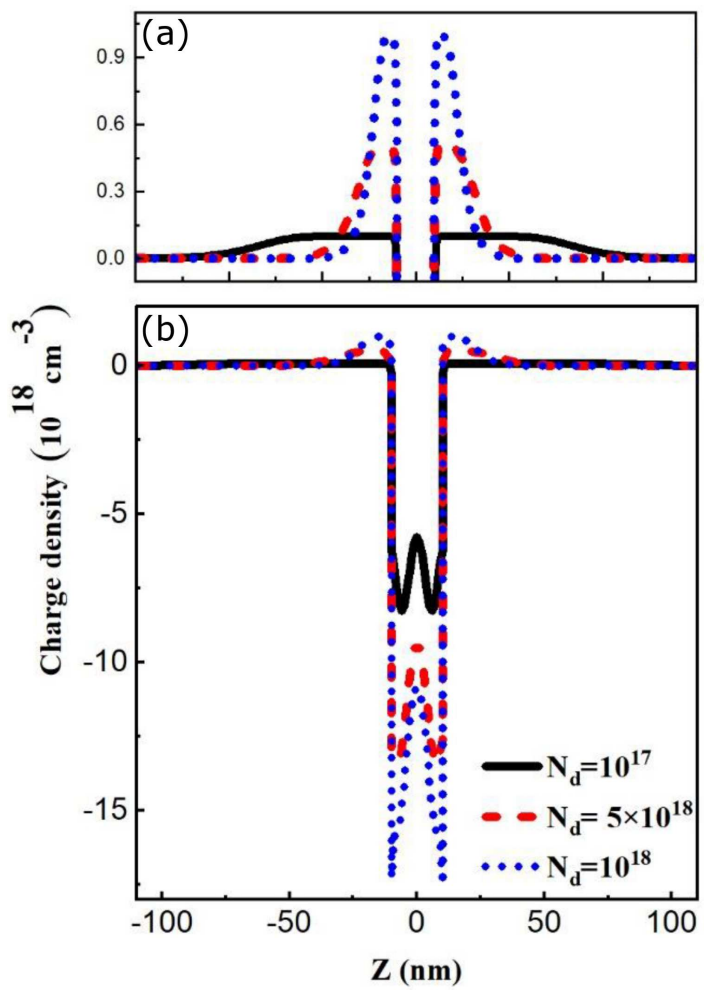

Fig. 4. The effect of donor concentration (in $\mathrm{cm}^{-3}$ ) of the cylinder on the distribution of charge density (a) outside and (b) inside of the QD along the cylinder axis. The charge density is in the range of $(0-1) \times 10^{18} \mathrm{~cm}^{-3}$.

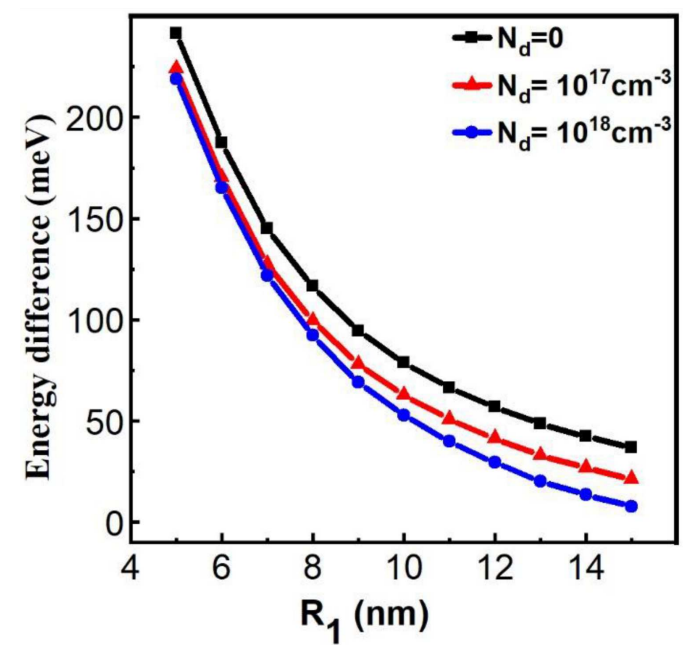

Fig. 5. The energy difference between ground and first excited states as a function of QD radius $R_{1}$.

weakens the effect of confinement. In other words, a larger transition energy exists in smaller spherical dots due to stronger quantum confinement.

The linear $\alpha^{(1)}$, nonlinear $\alpha^{(3)}$ and total absorption coefficients $\alpha$ of the structure with different doping concentrations are compared with the results of absorption coefficients without doping in Fig. 6. The resonance peak position experiences 


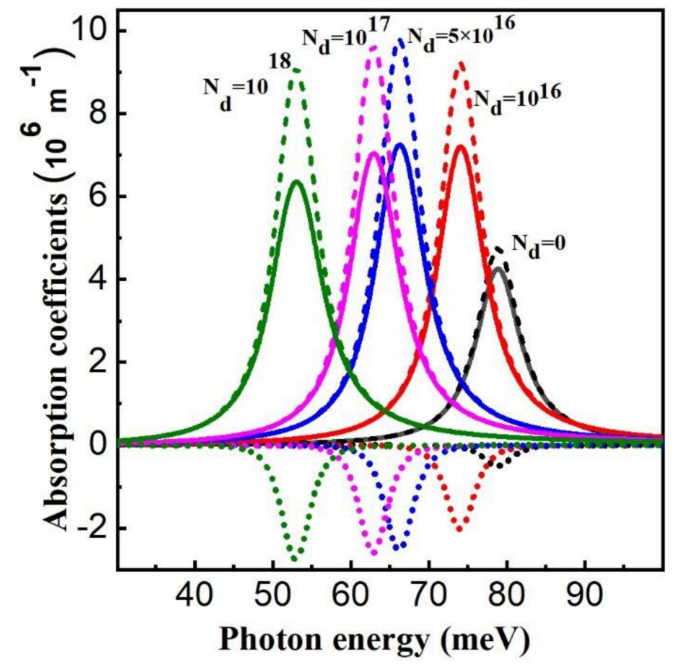

Fig. 6. The linear (dash line) $\alpha^{(1)}$, nonlinear (dot line) $\alpha^{(3)}$ and total absorption coefficients (solid line) $\alpha$ for the given structure at different doping concentrations $\left(\mathrm{cm}^{-3}\right)$.

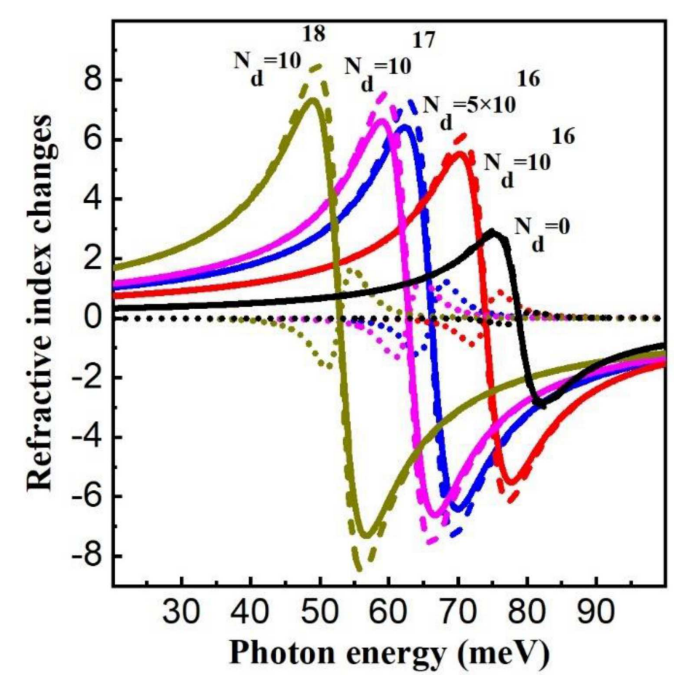

Fig. 7. The linear (dash line) $\Delta N^{(1)} / n_{r}$, nonlinear (dot line) $\Delta N^{(3)} / n_{r}$ and total refractive index changes (solid line) $\Delta N / n_{r}$ for the given structure based on GaAs/InAs interface in different doping concentrations $\left(\mathrm{cm}^{-3}\right)$.

a red shift by increasing the doping concentration. As it can be seen, the magnitude of the third order nonlinear absorption coefficient increases, just as the doping concentration does. When the doping concentration increases, the linear absorption coefficient increases as long as the concentration is $\leq 5 \times 10^{16} \mathrm{~cm}^{-3}$. When it is higher than this concentration, it begins to decrease.

There is a reason for these changes. According to (16), the nonlinear absorption coefficient is proportional to $M_{21}^{4}$, and since the overlapping of the wave functions increases just as concentrations do, the coefficient $\alpha^{(3)}$ increases too. In other words, the linear term is proportional to $M_{21}^{2} E_{21}$ (see (15)),

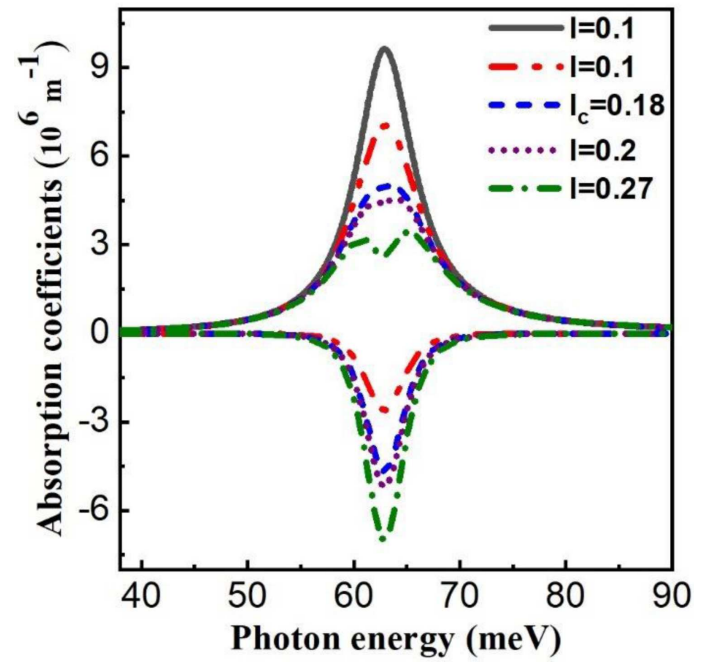

Fig. 8. The linear (solid line), nonlinear (negative) and total absorption coefficients as a function of photon energy for different incident optical intensities $\left(\mathrm{MW} / \mathrm{cm}^{2}\right)$ and $N_{d}=10^{17} \mathrm{~cm}^{-3}$.

and by increasing the doping concentration the term of $M_{21}^{2}$ increases, whereas the energy difference $E_{21}$ decreases. Nonetheless, at low concentrations the increase in $M_{21}$ overtakes the decrease of $E_{21}$, so that the linear term increases while in a higher concentration, $N_{d}>5 \times 10^{16} \mathrm{~cm}^{-3}$, the decrease in $E_{21}$ dominates the increase in $M_{21}$. Thus, the magnitude of the linear absorption coefficient peak decreases. The same behavior occurs in the total absorption as in the linear absorption due to the domination of linear term in $\alpha$ relation (17).

The magnitude of the resonance peaks of the linear, nonlinear and total refractive index changes as a function of photon energy for different doping concentrations - is shown in Fig. 7.

The magnitudes of the linear $\Delta N^{(1)} / n_{r}$ and the nonlinear refractive index changes $\Delta N^{(3)} / n_{r}$ are proportional to $M_{21}^{2}$ and $M_{21}^{4}$, respectively. As explained above, the magnitude of their peaks increases simultaneously as the donor concentration is increased.

Another parameter that affects changes in the magnitude of optical coefficients is the incident optical intensity $I$. The linear, nonlinear and total absorption coefficients against photon energy for different incident optical intensities are plotted in Fig. 8 for $N_{d}=10^{17} \mathrm{~cm}^{-3}$ and in Fig. 9 for $N_{d}=10^{16} \mathrm{~cm}^{-3}$. As the incident optical intensity increases, the nonlinear part of the absorption coefficient increases but the linear term does not change, as shown in (15)-(19). Since the linear and nonlinear terms have the opposite sign, the total absorption coefficient is reduced. As seen in Figs. 8 and 9 for $I>I_{c}$, the peak in the maximum of the total absorption is converted to two peaks. This effect occurs because the nonlinear term is dominated. 


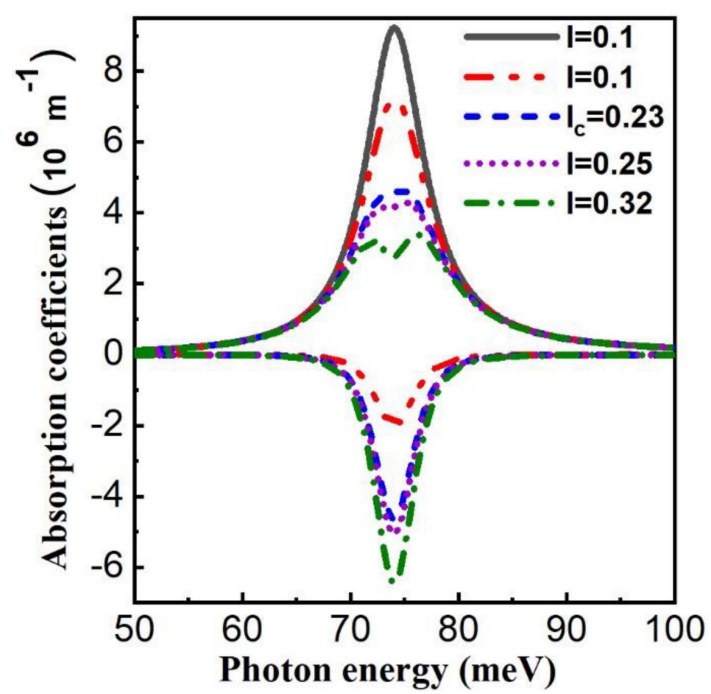

Fig. 9. The linear (solid line), nonlinear (negative) and total absorption coefficients as a function of photon energy for different incident optical intensities $\left(\mathrm{MW} / \mathrm{cm}^{2}\right)$ and $N_{d}=10^{16} \mathrm{~cm}^{-3}$.

\section{Conclusions}

The semiconductor heterostructure involving an InAs spherical quantum dot located at the center of an $n$-type doped GaAs cylindrical nanowire was investigated. The self-consistent solution of Schrödinger-Poisson equations in cylindrical coordinates was used to obtain the electronic structure, energy levels and optical properties of QD at different donor concentrations $N_{d}$ of the surrounding media. We found that as the doping concentration increases, the depletion region around QD decreases, and the conduction band bending becomes sharper. Moreover, a wider depletion region at a lower doping concentration leads to a larger change in electrostatic potential. The concentration of the positive and negative charges confirms the behavior of electrostatic potential. The energy difference between the lowest two levels decreases as the radius of QD increases but in higher doping concentrations, this difference becomes smaller. It is also deduced that the doping concentration of the surrounding media can play an important role in designing the optical properties of QD. The magnitude of the resonance peak of absorption coefficients and the refractive index changes depend on the doping concentration. As the doping concentration increases, the total absorption coefficient rises for $N_{d} \leq 5 \times 10^{16} \mathrm{~cm}^{-3}$ and then it decreases but the refractive index changes increase regardless. In both cases, they experience a red shift. Finally, when the incident optical intensity increases, the total absorption coefficients decrease. It seems that this behavior is due to the existence of a more mobile charge in the system. In conclusion, it is possible to design a quantum dot with the required electronic and optical properties in the far infra-red spectra.

\section{References}

[1] D. Stichtenoth, K. Wegener, C. Gutsche, I. Regolin, F.J. Tegude, W. Prost, M. Seibt, C. Ronning, Appl. Phys. Lett. 92, 163107 (2008).

[2] M. Henrik, A. Nicklas, S. Zhipei, L. Harri, Nanophotonics 8, 747 (2019).

[3] D. Dalacu, K. Mnaymneh, X. Wu, J. Lapointe, G.C. Aers, P.J. Poole, R.L. Williams, Appl. Phys. Lett. 98, 251101 (2011).

[4] M.J. Holmes, K. Choi, S. Kako, M. Arita, Y. Arakawa, Nano Lett. 14, 982 (2014).

[5] I. Friedler, C. Sauvan, J.P. Hugonin, P. Lalanne, J. Claudon, J.M. Gérard, Opt. Express 17, 2095 (2009).

[6] A. V. Maslov, M. I. Bakunov, C.-Z. Ning, J. Appl. Phys. 99, 024314 (2006).

[7] I. Ali, J. Jianliang, E. Deborah, Y. Muhammad, in: Proc. Int. Conf. on Optical Instruments and Technology: Micro/Nano Photonics: Materials and Devices, Beijing 2017, SPIE, 2017.

[8] B. Ilahi, M. Souaf, M. Baira, J. Alrashdi, L. Sfaxi, A. Alhazaa, H. Maaref, J. Nanomater. 2015, 847018 (2015).

[9] T. Yamauchi, M. Tabuchi, A. Nakamura, Appl. Phys. Lett. 84, 3834 (2004).

[10] L. Liang, W. Xie, Phys. B Condens. Matter 462, 15 (2015).

[11] L.-H. Peng, y. Hsu, C.W. Chuang, IEEE J. Sel. Top. Quantum Electron. 5, 756 (1999).

[12] C. Ronning, P.X. Gao, Y. Ding, Z.L. Wang, D. Schwen, Appl. Phys. Lett. 84, 783 (2004).

[13] S.J. Pearton, D.P. Norton, F. Ren, Small 3, 1144 (2007).

[14] R. Dingle, H. Störmer, A. Gossard, W. Wiegmann, Appl. Phys. Lett. 33, 665 (1978).

[15] L.D. Nguyen, L.E. Larson, U.K. Mishra, Proc. IEEE 80, 494 (1992).

[16] J. Sanchez-Dehesa, F. Meseguer, F. Borondo, J.C. Maan, Phys. Rev. $B$ 36, 5070 (1987).

[17] K.T. Kim, S.S. Lee, S. Chuang, J. Appl. Phys. 69, 6617 (1991).

[18] X. Wen, L.V. Dao, P. Hannaford, S. Mokkapati, H.H. Tan, C. Jagadish, Eur. Phys. J. B 62, 65 (2008).

[19] L.C. West, S.J. Eglash, Appl. Phys. Lett. 46, 1156 (1985).

[20] F. Ungan, E. Kasapoglu, H. Sarı, I. Sökmen, Superlattice. Microst. 46, 864 (2009). 
[21] F. Ungan, E. Öztürk, Y. Ergün, I. Sökmen, Superlattice. Microst. 41, 22 (2007).

[22] F. Ungan, J.C. Martínez-Orozco, R.L. Restrepo, M.E. Mora-Ramos, C.A. Duque, Superlattice. Microst. 126, 89 (2019).

[23] S. Wang, Y. Kang, X. Li, J. Semicond. 37, 112001 (2016).

[24] E. Öztürk, Cumhuriyet Sci. J. 39, 720 (2018).

[25] V. Albe, L.J. Lewis, Phys. B Condens. Matter 301, 233 (2001).

[26] S. Ünlü, İ. Karabulut, H. Şafak, Phys. E Low Dimens. Syst. Nanostruct. 33, 319 (2006).

[27] W. Xie, Physica B Condens. Matter 403, 4319 (2008).

[28] N. Aghoutane, M. El-Yadri, A. El Aouami, E. Feddi, G. Long, M. Sadoqi, F. Dujardin, C. V. Nguyen, N.N. Hieu, H.V. Phuc, MRS Commun. 9, 663 (2019).

[29] N. Aghoutane, M. El-Yadri, A. Aouami, E. Feddi, F. Dujardin, M. El haouari, C. Duque, C.V. Nguyen, H.V. Phuc, Appl. Phys. A 125, 17 (2018).

[30] J.C. Martínez-Orozco, K.A. Rodríguez-Magdaleno, J.R. Suárez-López, C.A. Duque, R.L. Restrepo, Superlattice. Microst. 92, 166 (2016).
[31] M. Baira, B. Salem, N. Madhar, B. Ilahi, Nanomaterials 9, 124 (2019).

[32] M. Gambhir, V. Prasad, Rev. Mex. de Fis. 64, 439 (2018).

[33] C. Cetinkaya, E. Cokduygulular, F. Nutku, O. Donmez, J. Puustinen, J. Hilska, A. Erol, M. Guina, J. Alloys Compd. 739, 987 (2018).

[34] G. Safarpour, M. Barati, M. Vahdani, Phys. E Low Dimens. Syst. Nanostruct. 44, 728 (2011).

[35] G. Safarpour, M. Barati, J. Lumin. 137, 98 (2013).

[36] G. Bastard, Wave Mechanics Applied to Semiconductor Heterostructures, John Wiley and Sons Inc, United States 1990.

[37] I.H. Tan, G.L. Snider, L.D. Chang, E.L. Hu, J. Appl. Phys. 68, 4071 (1990).

[38] M. Karimi, G. Rezaei, J. Appl. Phys. 111, 064313 (2012).

[39] K.A. Rodríguez-Magdaleno, J.C. Martínez-Orozco, I. Rodríguez-Vargas, M.E. Mora-Ramos, C.A. Duque, J. Lumin. 147, 77 (2014).

[40] M. Califano, P. Harrison, Phys. Rev. B 61, 10959 (2000). 